\title{
Feature Isolation for Hypothesis Testing in Retinal Imaging: An Ischemic Stroke Prediction Case Study
}

\author{
Gilbert Lim, ${ }^{1}$ Zhan Wei Lim, ${ }^{1}$ Dejiang Xu, ${ }^{1}$ Daniel S.W. Ting, ${ }^{2}$ \\ Tien Yin Wong, ${ }^{2}$ Mong Li Lee, ${ }^{1}$ Wynne Hsu ${ }^{1}$ \\ ${ }^{1}$ School of Computing, National University of Singapore \\ ${ }^{2}$ Singapore Eye Research Institute, Singapore National Eye Centre, Duke-NUS Medical School
}

\begin{abstract}
Ischemic stroke is a leading cause of death and long-term disability that is difficult to predict reliably. Retinal fundus photography has been proposed for stroke risk assessment, due to its non-invasiveness and the similarity between retinal and cerebral microcirculations, with past studies claiming a correlation between venular caliber and stroke risk. However, it may be that other retinal features are more appropriate. In this paper, extensive experiments with deep learning on six retinal datasets are described. Feature isolation involving segmented vascular tree images is applied to establish the effectiveness of vessel caliber and shape alone for stroke classification, and dataset ablation is applied to investigate model generalizability on unseen sources. The results suggest that vessel caliber and shape could be indicative of ischemic stroke, and sourcespecific features could influence model performance.
\end{abstract}

\section{Introduction}

Ischemic stroke is an emergency medical condition characterized by a reduction of blood supply to the brain due to a blocked artery. Stroke is the fifth-leading cause of death in the United States, accounting for over 5\% of deaths, and is a leading cause of long-term disability (Benjamin et al. 2018). While there exist evidence-based guidelines for assessing stroke risk, many of the recommendations involve specialized tests, with general population-level genetic screening currently not recommended.

Given the widespread prevalence of ischemic stroke and the relative inability to predict its occurrence as of the present, it is tempting to explore other modalities for purposes of stroke risk assessment. One such modality is retinal imaging, which has the attraction of being a noninvasive and cost-effective procedure that has the potential to be broadly deployed. Further, previous studies have observed correlations between vascular features and incidence of stroke (Baker et al. 2010). With the recent successes of deep learning in medical imaging classification tasks (Litjens et al. 2017), it is only natural to ask whether this model paradigm might also be applicable to stroke prediction.

An enduring criticism of deep neural network models has however been their lack of interpretability, which is of particular concern for clinical tasks (Caruana et al. 2015).

Copyright (c) 2019, Association for the Advancement of Artificial Intelligence (www.aaai.org). All rights reserved.
Stroke risk prediction, as opposed to general risk factor prediction, poses an additional difficulty in that images for positive cases are hard to come by. In particular, for the purposes of this study, patients and images positive for ischemic stroke could only be drawn from a single source, the MultiCentre Retinal Stroke (MCRS) study (De Silva et al. 2009). This rarity of condition-specific data is not uncommon in the medical domain. Negative data was relatively abundant, and could be obtained from multiple sources.

However, this naturally raises a question of the trained models: did they actually learn what they were expected to (stroke vs. non-stroke), or did they learn something incidental? Put another way, there may be some combination of features (e.g. ethnicity, camera characteristics, compression artifacts, etc) that coincide with the stroke-positive/negative divide in the training data. If so, it is plausible that the model will instead learn these non-stroke-relevant features, especially if they happen to be more discriminative to the deep neural network architecture. Specifically, for this stroke prediction task, the source domain involves labeled negative data from multiple datasets, but labeled positive data from just a single dataset. However, the desired target domain would involve the classification of data from multiple datasets in general. Since the source and target domains are not truly the same, a classifier trained on the source domain may not fully apply to data from the target domain. This problem is known as domain shift in machine learning (Quiñonero-Candela et al. 2009).

A standard response to domain shift would be to perform domain adaptation, for example through preprocessing or transferring statistics between the source and target domains (Sun, Feng, and Saenko 2016). However, in this case, a proper evaluation of domain adaptation is not possible, because of the lack of even unlabeled positive data in the target domain. As such, we set out to estimate possible upper and lower performance bounds on the stroke risk prediction task, by performing dataset ablation, to examine the sensitivity of model performance to variance in the training datasets.

Additionally, saliency map visualizations may also be examined to verify that the model is working on plausible features, as was done for (Poplin et al. 2018). Here, we additionally practise feature isolation, in which only the most relevant data according to the literature, the vascular tree, is retained. A similar idea has recently been reported for stan- 
dardizing optical coherence tomography images from different sources, before performing classification on the abstracted segmentation map (De Fauw et al. 2018).

We believe that this experimental methodology addresses two issues that may not have been fully appreciated in the literature. First, the performance of machine learning models on out-of-set data is often not fully explored. In particular, the contribution of different data subsets within the training data may not be comprehensively analyzed. Standard crossvalidation for example attempts to assess model generalizability, but does not realistically model true out-of-set data, since training data is collated from all sources (i.e. validation data for each cross-validation fold already contains data from all sources). A more conservative estimate of model generalizability is therefore obtained by explicitly training and validating models on non-overlapping data sources.

Second, the precise attribution of clinical decision is extremely important, for clinical decision support systems to be accepted (Shortliffe and Sepúlveda 2018). Existing endto-end deep neural network architectures have been widely criticized as black boxes possibly exhibiting unexpected behaviour, as in the case of adversarial images. One workable approach to counter this would then be to first identify and isolate relevant features - in this case the retinal vascular tree - considering domain knowledge, and utilize them as auditable intermediate goals (Lim, Hsu, and Lee 2018). This guarantees that model performance is obtained only from the isolated features, rather than merely retrospectively claimed to be such from saliency map analysis.

This case study on stroke risk prediction describes an attempt to mitigate interpretability and generalizability issues by constraining the input information available to the models, and in this manner aims to offer insights to the challenge of establishing generalizable results in real-world medical prediction tasks (Trucco et al. 2013).

\section{Related Work}

There exists a rich literature on the association of retinal blood vessel features with stroke risk, motivated by similarities between retinal and cerebral microcirculations. Previous studies have generally tended towards the extraction of intermediate summary features from retinal photographs, such as vessel caliber and nicking (Baker et al. 2010), with a meta-analysis suggesting that wider retinal venular caliber is related to increased risk of stroke (McGeechan et al. 2009). Such studies depend on vessel type (artery/vein) being identified for the major vessels (Xu et al. 2018), and their measurements taken with specialized software (Lau et al. 2014) for further statistical analysis.

However, it remains uncertain whether these handpicked summary features are in fact the most suitable for the stroke risk prediction task. End-to-end deep learning attempts to automate the intermediate feature selection process, and has usually been demonstrated to yield superior performance as compared to handpicked features. Deep neural network models applied to retinal imaging have exhibited performance comparable to human graders in classifying for conditions such as referable diabetic retinopathy (Gulshan et al.
2016; Ting et al. 2017), as well as age-related macular degeneration and glaucoma (Ting et al. 2017).

An important distinction between the abovementioned classification tasks, and the stroke prediction task described here, is that the classification tasks could be performed by human graders. In each case, there existed well-established visual grading guidelines against which human graders could be trained and assessed. Therefore, it is known that a working model should be attainable in principle, if only by reproducing the human procedure. For example, since diabetic retinopathy is graded based on the presence and quantity of lesions such as microaneurysms, haemorrhages and exudates, each with their own distinct appearance, much earlier work had been devoted to individually identifying and counting such lesions, before applying the specified guidelines (Niemeijer et al. 2010; Lim et al. 2014).

There is in contrast no such assurance for stroke prediction, from the inability of human experts to reliably diagnose stroke from retinal photographs, as far as is known. The closest work on this front is then on the prediction of cardiovascular risk factors from retinal photographs (Poplin et al. 2018), where it was unknown beforehand whether it was possible to deduce risk factors such as age, gender, smoking status and body mass index solely from retinal photographs. Using a development set - of over 1.7 million images - and a clinical validation set drawn from the same two sources (UK Biobank and EyePACS), the authors discovered near-perfect predictive ability for gender, and significantly better-thanbaseline predictions for most other targeted risk factors.

\section{Methodology}

Figure 1 summarizes the preprocessing that we carried out to standardize the input to the models. All images had their central circular portion, corresponding to the actual retina, detected and mapped to a $512 \times 512$ pixel template.

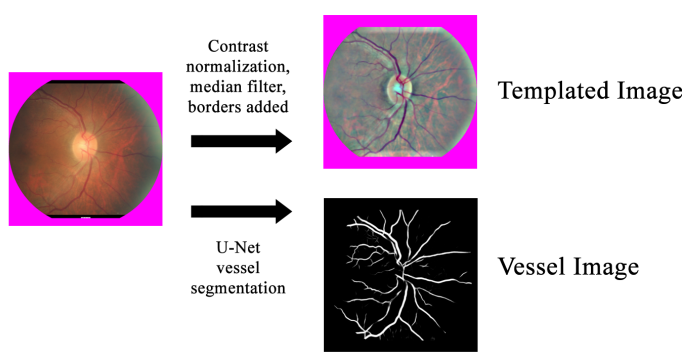

Figure 1: Retinal image preprocessing procedure

We mitigate for possible differences in surface appearance between the datasets with contrast normalization. A median filter with kernel width of 5 pixels was then applied to remove high-frequency camera noise and source-specific features, which might otherwise help to distinguish between the dataset sources. A top and bottom border of 40 pixels was then applied to standardize the presence of borders from the original images. These are referred to as templated images.

In addition, to investigate whether ischemic stroke could be predicted solely based on blood vessel data, we segment 
the vascular tree (vessel mask) with a U-Net implementation (Orobix 2016; Ronneberger, Fischer, and Brox 2015). These are referred to as vessel images.

Model Architecture. A 19-layer VGGNet architecture (Simonyan and Zisserman 2015) as described in (Ting et al. 2017) was used for all experiments, with the exception of the output layer now consisting of two nodes, representing the stroke-positive and stroke-negative class respectively. As such, the predicted value of the stroke-positive output node, given an input image, can be interpreted as some measure of stroke risk for the patient corresponding to that image. The models were trained with a batch size of 32, base learning rate of 0.001 and momentum of 0.9 , with a step decay of 0.98 every 1000 iterations. Xavier initialization (Glorot and Bengio 2010) of node weights was used, with preliminary experiments suggesting that model performance is robust as to model architecture (e.g. ResNet), usage of pretrained model weights and training parameters.

Datasets. Table 1 shows the six retina image datasets used. Images positive for ischemic stroke were obtained from the MCRS study, which consisted of patients from Singapore, Sydney and Melbourne. No negative images were available from this study. Images negative for ischemic stroke were drawn from five datasets: the Singapore Chinese Eye Study dataset (SCES), the Singapore Malay Eye Study dataset (SiMES), the Singapore Indian Eye Study dataset (SiNDI), the Diabetic Management Programme (Melbourne) dataset (DMPMelb) and the Singapore Prospective Study Program dataset (SP2). The number of negative images was set to roughly match that of the available positive images. All images thus selected were then filtered for being optic disc (OD) centered, to ensure presence of the major vessels.

\begin{tabular}{r|c|c|c|c}
\hline Dataset & \multicolumn{2}{|c|}{ templated } & \multicolumn{2}{c}{ matched } \\
\hline & \#images & $\begin{array}{c}\text { vessel pixels } \\
\text { mean (std. } \\
\text { dev) }\end{array}$ & \#images & $\begin{array}{c}\text { vessel pixels } \\
\text { mean (std. } \\
\text { dev) }\end{array}$ \\
\hline \multicolumn{4}{c}{ Stroke-positive } \\
\hline MCRS & 4528 & $10127(6872)$ & 972 & $9971(1492)$ \\
\hline \multicolumn{5}{|c}{ Stroke-negative } \\
\hline SCES & 1751 & $15160(5959)$ & 268 & $10403(1375)$ \\
\hline SiMES & 1488 & $15954(6542)$ & 188 & $10231(1414)$ \\
\hline SiNDI & 1662 & $15199(6311)$ & 238 & $10272(1396)$ \\
\hline DMPMelb & 715 & $12156(6337)$ & 165 & $10207(1380)$ \\
\hline SP2 & 1006 & $13735(5360)$ & 206 & $10140(1412)$ \\
\hline
\end{tabular}

Table 1: Datasets Summary

We observe that there is a wide variance in the appearance of the vessel images in terms of thresholded vessel mask density, possibly due to differences between the dataset images and the images that the U-Net segmentation model was trained on. As such, a subset of each dataset was defined such that the distribution of vessel mask pixels was similar, by filtering for only vessel images with $10000 \pm 2500$ vessel mask pixels each. These subsets are referred to as the matched subsets of each dataset.

\section{Experiments}

For each dataset, we split the images into a development set, and a test set. The development set is itself split into a training set of images that are shown to the model, and a validation set that is used to derive early stopping and operating point thresholds. Images were randomly assigned to the training, validation and test sets, subject to the constraint that there is no overlap at patient level. The distribution of images is approximately $80 \%$ for the training set, $5 \%$ for the validation set, and $15 \%$ for the test set.

Three sets of experiments were carried out on four types of input images: all templated images (TA), templated images matched for vessel mask pixels (TM), all vessel images (VA) and and vessel images matched for vessel mask pixels (VM). Data augmentation was implemented through the random flipping and rotation of input images.

The first set of experiments (E-All) used all six datasets. During training, stroke-positive and stroke-negative images were sampled in equal proportion. Model performance in terms of AUC was evaluated on the validation set every 500 iterations, and early stopping applied to limit overfitting once marginal improvement (a change in validation AUC of magnitude less than 0.005) had been observed over the previous iterations. A model operating point threshold was then picked using model performance on the validation set to balance sensitivity and specificity. The models were then evaluated on the test sets.

The second set of experiments (E-Split1) drew negativestroke training and validation images from only SCES, SiMES and SiNDI datasets, whereas the third set of experiments (E-Split2) drew negative-stroke training and validation images from only DMPMelb and SP2 datasets. These negative-stroke subsets were chosen based on their similar vessel pixel distributions. Both E-Split1 and E-Split2 used stroke-positive images from the MCRS dataset.

\section{Performance of Model for Stroke Prediction}

Figure 2 shows the ROC curves for the respective models obtained in the 3 sets of experiments. We observe that the model from the first set of experiments trained on the templated images (E-All-TA) gives the best AUC.

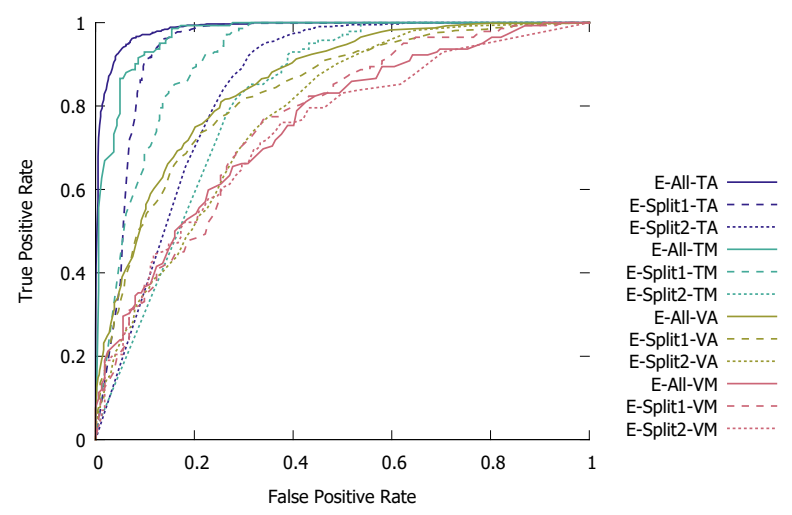

Figure 2: ROC curves for respective models 


\begin{tabular}{|c|c|c|c|c|c|c|c|c|c|c|c|c|}
\hline & & & & & & & & & & & & \\
\hline Exp & Type & All & Split1 & Split2 & SCES & SiMES & SiNDI & DMPMelb & SP2 & Validation & Iter & $\alpha$ \\
\hline \multirow{10}{*}{ E-All } & \multirow[b]{2}{*}{ TA } & 0.972 & 0.972 & 0.972 & 0.972 & 0.972 & 0.972 & 0.972 & 0.972 & 0.921 & \multirow[b]{2}{*}{14000} & \multirow[b]{2}{*}{0.43} \\
\hline & & $\begin{array}{c}0.890 \\
(0.987)\end{array}$ & $\begin{array}{c}0.893 \\
(0.986)\end{array}$ & $\begin{array}{c}0.880 \\
(0.986)\end{array}$ & $\begin{array}{c}0.903 \\
(0.988)\end{array}$ & $\begin{array}{c}1.000 \\
(0.998)\end{array}$ & $\begin{array}{c}0.783 \\
(0.970)\end{array}$ & $\begin{array}{c}0.703 \\
(0.966)\end{array}$ & $\begin{array}{c}1.000 \\
(0.996)\end{array}$ & $\begin{array}{c}0.923 \\
(0.978)\end{array}$ & & \\
\hline & \multirow{3}{*}{$\mathrm{TM}$} & 0.993 & 0.993 & 0.993 & 0.993 & 0.993 & 0.993 & 0.993 & 0.993 & 0.840 & \multirow{3}{*}{11000} & \multirow{3}{*}{0.30} \\
\hline & & 0.827 & 0.779 & 0.914 & 0.789 & 0.931 & 0.649 & 0.808 & 1.000 & 0.821 & & \\
\hline & & $(0.970)$ & $(0.961)$ & $(0.978)$ & $(0.947)$ & $(0.976)$ & $(0.936)$ & $(0.952)$ & $(0.984)$ & $(0.864)$ & & \\
\hline & \multirow{3}{*}{ VA } & 0.818 & 0.818 & 0.818 & 0.818 & 0.818 & 0.818 & 0.818 & 0.818 & 0.667 & \multirow{3}{*}{15500} & \multirow{3}{*}{0.35} \\
\hline & & 0.729 & 0.760 & 0.641 & 0.681 & 0.955 & 0.662 & 0.406 & 0.800 & 0.663 & & \\
\hline & & $(0.855)$ & $(0.869)$ & $(0.811)$ & $(0.826)$ & $(0.960)$ & $(0.828)$ & $(0.667)$ & $(0.906)$ & $(0.750)$ & & \\
\hline & \multirow[b]{2}{*}{ VM } & 0.753 & 0.753 & 0.753 & 0.753 & 0.753 & 0.753 & 0.753 & 0.753 & 0.700 & \multirow[b]{2}{*}{6000} & \multirow[b]{2}{*}{0.45} \\
\hline & & $\begin{array}{c}0.611 \\
(0.754)\end{array}$ & $\begin{array}{c}0.615 \\
(0.751)\end{array}$ & $\begin{array}{c}0.603 \\
(0.749)\end{array}$ & $\begin{array}{c}0.421 \\
(0.627)\end{array}$ & $\begin{array}{c}0.828 \\
(0.847)\end{array}$ & $\begin{array}{c}0.649 \\
(0.777)\end{array}$ & $\begin{array}{c}0.423 \\
(0.655)\end{array}$ & $\begin{array}{c}0.750 \\
(0.811)\end{array}$ & $\begin{array}{c}0.643 \\
(0.730)\end{array}$ & & \\
\hline \multirow{12}{*}{ E-Split1 } & \multirow{3}{*}{ TA } & 0.973 & 0.973 & 0.973 & 0.973 & 0.973 & 0.973 & 0.973 & 0.973 & 0.912 & \multirow{3}{*}{18500} & \multirow{3}{*}{0.22} \\
\hline & & 0.842 & 0.921 & 0.614 & 0.926 & 1.000 & 0.842 & 0.079 & 0.973 & 0.926 & & \\
\hline & & $(0.940)$ & $(0.990)$ & $(0.796)$ & $(0.991)$ & (0.994) & $(0.978)$ & $(0.496)$ & $(0.994)$ & $(0.966)$ & & \\
\hline & \multirow{3}{*}{$\mathrm{TM}$} & 0.965 & 0.965 & 0.965 & 0.965 & 0.965 & 0.965 & 0.965 & 0.965 & 0.860 & \multirow{3}{*}{9000} & \multirow{3}{*}{0.52} \\
\hline & & 0.741 & 0.798 & 0.638 & 0.816 & 0.897 & 0.703 & 0.192 & 1.000 & 0.829 & & \\
\hline & & $(0.914)$ & $(0.950)$ & $(0.849)$ & $(0.950)$ & $(0.972)$ & (0.914) & $(0.662)$ & $(0.984)$ & $(0.891)$ & & \\
\hline & \multirow{3}{*}{ VA } & 0.819 & 0.819 & 0.819 & 0.819 & 0.819 & 0.819 & 0.819 & 0.819 & 0.681 & \multirow{3}{*}{7000} & \multirow{3}{*}{0.45} \\
\hline & & 0.696 & 0.760 & 0.510 & 0.739 & 0.799 & 0.746 & 0.307 & 0.647 & 0.678 & & \\
\hline & & (0.833) & $(0.862)$ & $(0.746)$ & $(0.841)$ & $(0.902)$ & $(0.846)$ & $(0.628)$ & $(0.823)$ & $(0.733)$ & & \\
\hline & \multirow{3}{*}{ VM } & 0.810 & 0.810 & 0.810 & 0.810 & 0.810 & 0.810 & 0.810 & 0.810 & 0.720 & \multirow{3}{*}{14500} & \\
\hline & & 0.574 & 0.625 & 0.483 & 0.316 & 1.000 & 0.649 & 0.269 & 0.656 & 0.657 & & 0.55 \\
\hline & & $(0.759)$ & $(0.795)$ & $(0.685)$ & $(0.650)$ & $(0.941)$ & $(0.802)$ & $(0.516)$ & $(0.808)$ & $(0.722)$ & & \\
\hline & & 0.995 & 0.995 & 0.995 & 0.995 & 0.995 & 0.995 & 0.995 & 0.995 & 0.968 & & \\
\hline & TA & 0.485 & 0.345 & 0.884 & 0.117 & 0.960 & 0.017 & 0.792 & 0.947 & 0.988 & 7500 & 0.46 \\
\hline E-Snlit2 & & $(0.846)$ & $(0.798)$ & (0.984) & $(0.830)$ & $(0.997)$ & $(0.575)$ & $(0.974)$ & $(0.983)$ & $(0.992)$ & & \\
\hline E-Split 2 & & 0.979 & 0.979 & 0.979 & 0.979 & 0.979 & 0.979 & 0.979 & 0.979 & 0.960 & & \\
\hline & $\mathrm{TM}$ & 0.463 & 0.250 & 0.845 & 0.053 & 0.793 & 0.027 & 0.885 & 0.813 & 1.000 & 7000 & 0.35 \\
\hline & & $(0.815)$ & $(0.717)$ & $(0.983)$ & $(0.604)$ & $(0.961)$ & $(0.636)$ & $(0.970)$ & $(0.964)$ & $(0.955)$ & & \\
\hline & & 0.840 & 0.840 & 0.840 & 0.840 & 0.840 & 0.840 & 0.840 & 0.840 & 0.782 & & \\
\hline & VA & 0.579 & 0.508 & 0.785 & 0.362 & 0.924 & 0.275 & 0.584 & 0.920 & 0.798 & 16000 & 0.07 \\
\hline & & (0.779) & $(0.737)$ & $(0.897)$ & $(0.671)$ & $(0.957)$ & $(0.602)$ & $(0.806)$ & $(0.956)$ & $(0.870)$ & & \\
\hline & & 0.697 & 0.697 & 0.697 & 0.697 & 0.697 & 0.697 & 0.697 & 0.697 & 0.720 & & \\
\hline & VM & 0.673 & 0.615 & 0.776 & 0.421 & 0.931 & 0.568 & 0.654 & 0.875 & 0.714 & 17500 & 0.16 \\
\hline & & $(0.740)$ & (0.699) & $(0.806)$ & $(0.586)$ & $(0.873)$ & $(0.657)$ & $(0.724)$ & $(0.859)$ & $(0.778)$ & & \\
\hline
\end{tabular}

Table 2: Detailed Experimental Results. Sensitivity (first value), specificity (second value), and AUC (in bracket) are obtained using the corresponding threshold $\alpha$ for each model.

Dataset Ablation Analysis. Table 2 gives the complete experimental results. The E-All-TA model has the best performance with AUC $\geq 0.966$. The E-Split1-TA model has good performance on the held-out test images (AUC $=0.990$ ). However, performance on unseen images were mixed, with excellent performance on SP2 (AUC $=0.994)$ but poor performance on DMPMelb (AUC $=0.496$ ). One explanation could be SP2 is a Singapore-based source, the same as SCES, SiMES and SiNDI. As such, the E-Split1-TA model learnt to classify the negative class based on the general features of the Singapore-based sources. Similarly, the ESplit2-TA model gives good performance on the held-out test images with AUC $=0.984$, but performance on unseen sources were mixed: SCES (AUC $=0.830$ ), SiMES (AUC

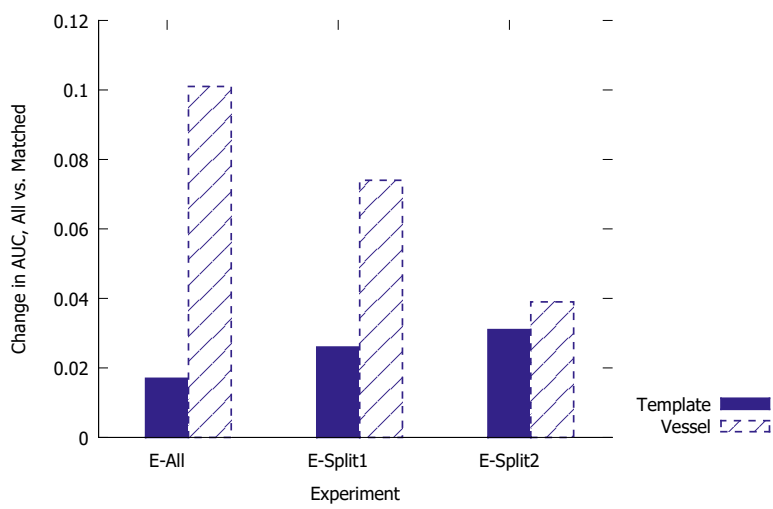

Figure 3: Effect of feature isolation on AUC
$=0.997)$ and $\operatorname{SiNDI}(\mathrm{AUC}=0.575)$.

Feature Isolation Analysis. We minimize the possible confounding influence of source-specific features by performing feature isolation to segment the vessels. We observe that the performances in the E-All experiments are consistent across all datasets. For the models trained using different image types, the overall performance was reduced from an AUC $=0.987$ for $\mathrm{TA}$ to $\mathrm{AUC}=0.970$ for $\mathrm{TM}$, to $\mathrm{AUC}=0.855$ for VA, and finally to AUC $=0.754$ for VM.

Figure 3 shows the impact of feature isolation on AUC. Closer examination reveals that the performance loss for EAll-TM against E-All-TA was much smaller compared to

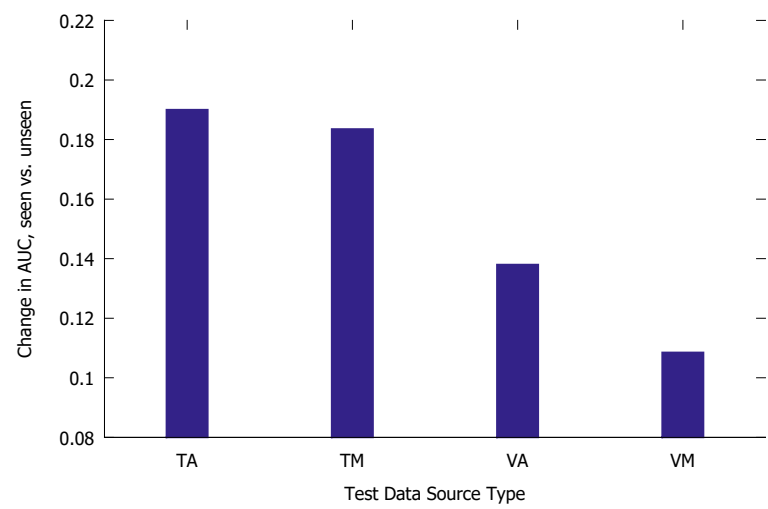

Figure 4: Performance difference on seen vs. unseen data, average of E-Split1 and E-Split2 


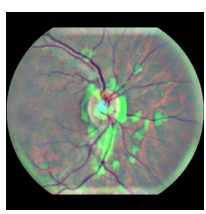

(a) E-All-TA

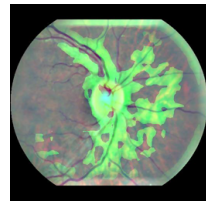

(e) E-Split1-TA

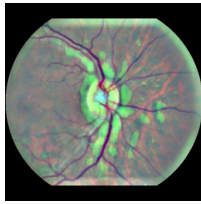

(i) E-Split2-TA

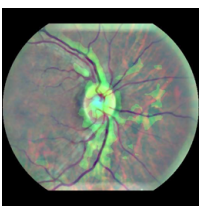

(b) E-All-TM

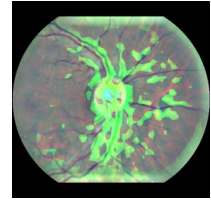

(f) E-Split1-TM

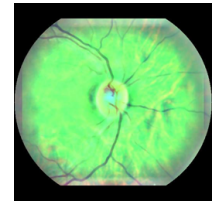

(j) E-Split2-TM

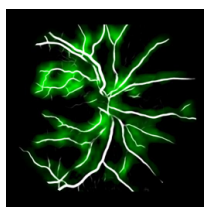

(c) E-All-VA

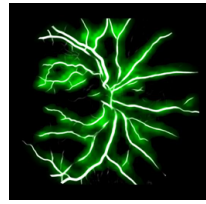

(g) E-Split1-VA

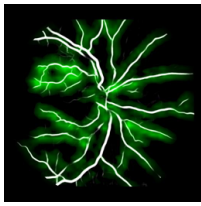

(k) E-Split2-VA

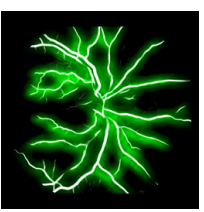

(d) E-All-VM

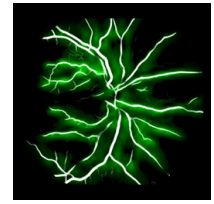

(h) E-Split1-VM

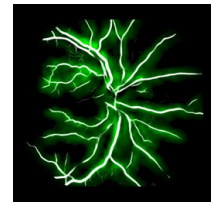

(1) E-Split2-VM

Figure 5: Example model visualizations

that between E-All-VM and E-All-VA, suggesting that the quantity of vessel pixels in the vessel images is indeed a distinguishing factor that should be normalized across datasets.

We also investigate the effect of feature isolation on the generalizability of these models. Figure 4 shows the change in AUC for seen vs. unseen data, for each set of experiments. A smaller change in AUC indicates better generalizability in terms of more stable model performance. In aggregate, using vessel images produces improved generalizability, as evidenced by the smaller values for VA/VM as compared to TA/TM. Of particular note is the performance for the SiNDI test set (see Table 2), which improved from 0.636 for TM, to 0.657 for VM, despite performance being expected to be poorer for VM compared to TM in general.

Overall, even under the feature isolation regime, the models managed to achieve an AUC of at least 0.685 for unseen sources (E-Split1-VM on Split2: AUC $=0.685$, ESplit2-VM on Split1: AUC $=0.699$ ), which supports the hypothesis that ischemic stroke might be indicated by vascular data alone. We confirm this by utilizing the gradientweighted class activation mapping (Grad-CAM) (Selvaraju et al. 2017) to visualize the saliency maps. Figure 5 shows that retinal vessel appearance is involved in distinguishing between classes.

\section{Discussion}

From the dataset ablation analysis, it was found that model classification performance on within-set negative data was consistently better than on out-of-set negative data, suggesting that the trained models may indeed be overfitting on characteristics unrelated to any actual features manifested by the mechanism of stroke risk. In other words, the templated image data may exhibit environment-specific differentiating features that significantly help to distinguish between the stroke-positive and stroke-negative classes.
From the feature isolation analysis, it was found that the segmented vascular tree alone remained predictive of stroke risk, as indicated by the medical literature. Raw classification performance dropped for vessel images as compared to template images, with the difference in performance plausibly attributable to the additional information contained by the template images. However, some of the features learnt from this additional information might be environmentspecific, and not condition-specific, and therefore spurious for stroke risk prediction. This is supported by the performance of vessel image models generally being more stable on out-of-set test data, in the sense of sensitivity/specificity being closer to being balanced at the validation threshold.

The major limitation of this study pertains to the nature of the datasets, in particular the availability of strokepositive images from only a single source. Much of the experimental effort has therefore been expended in either attempting to quantify its impact on model generalizability by dataset ablation, or attempting to compensate by minimizing the effect of non-critical appearance features with vessel images. This issue is exacerbated by the relatively small quantity of stroke-positive image data available. Previous major disease classification and risk factor prediction studies using deep neural network models on retinal images (Gulshan et al. 2016; Ting et al. 2017; Poplin et al. 2018) involved hundreds of thousands to millions of images, with large initial performance gains as the quantity of training images increased (Gulshan et al. 2016). In future work, we hope to more conclusively validate the possibility of stroke risk prediction using a wider variety of image data for each class, and against other methods.

\section{Conclusion}

In this paper, we have investigated the potential of deep neural network models to predict ischemic stroke, a task of con- 
siderable medical interest. Initial experiments were encouraging, and achieved excellent performance on the test set. However, due to the nature of the stroke-positive images being derived from a single source, feature isolation by vascular tree extraction and dataset ablation were attempted to quantify the influence of these factors on performance. It was found that model performance was not wholly generalizable to images from unseen sources, although vessel images alone remained discriminative of ischemic stroke.

\section{Acknowledgements}

This work was supported by grant R-252-000-A19-490.

\section{References}

Baker, M. L.; Hand, P. J.; Liew, G.; Wong, T. Y.; Rochtchina, E.; Mitchell, P.; Lindley, R. I.; Hankey, G. J.; Wang, J. J.; and the Multi-Centre Retinal Stroke Study Group. 2010. Retinal microvascular signs may provide clues to the underlying vasculopathy in patients with deep intracerebral hemorrhage. Stroke 41(4):618-623.

Benjamin, E. J.; Virani, S. S.; Callaway, C. W.; Chamberlain, A. M.; Change, A. R.; Cheng, S.; Chiuve, S. E.; Cushman, M.; Delling, F. N.; Deo, R.; de Ferranti, S. D.; Ferguson, J. F.; et al. 2018. Heart disease and stroke statistics - 2018 update: a report from the american heart association. Circulation 137(12):e67e492.

Caruana, R.; Lou, Y.; Gehrke, J.; Koch, P.; Sturm, M.; and Elhadad, N. 2015. Intelligible models for healthcare: Predicting pneumonia risk and hospital 30-day readmission. In ACM SIGKDD, 17211730 .

De Fauw, J.; Ledsam, J. R.; Romera-Paredes, B.; Nikolov, S.; Tomasev, N.; Blackwell, S.; Askham, H.; Glorot, X.; O’Donoghue, B.; Visentin, D; van den Driessche, G.; Lakshminarayan, B.; Meyer, C.; Mackinder, F.; Bouton, S.; et al. 2018. Clinically applicable deep learning for diagnosis and referral in retinal disease. Nature Medicine 24:1342-1350.

De Silva, D. A.; Liew, G.; Wong, M.-C.; Chang, H. M.; Chen, C.; Wang, J. J.; Baker, M. L.; Hand, P. J.; Rochtchina, E.; Liu, E. Y.; Mitchell, P.; Lindley, R. I.; and Wong, T. Y. 2009. Retinal vascular caliber and extracranial carotid disease in patients with acute ischemic stroke: the multi-centre retinal stroke (mcrs) study. Stroke 40(12):3695-3699.

Glorot, X., and Bengio, Y. 2010. Understanding the difficulty of training deep feedforward neural networks. In AISTATS, 249-256.

Gulshan, V.; Peng, L.; Coram, M.; Stumpe, M. C.; Wu, D.; Narayanaswamy, A.; Venugopalan, S.; Widner, K.; Madams, T.; Cuadros, J.; Kim, R.; Raman, R.; Nelson, P. C.; Mega, J. L.; and Webster, D. R. 2016. Development and validation of a deep learning algorithm for detection of diabetic retinopathy in retinal fundus photographs. JAMA 316(22):2402-2410.

Lau, Q. P.; Lee, M. L.; Hsu, W.; and Wong, T. Y. 2014. The Singapore eye vessel assessment system. Image Analysis and Modeling in Ophthalmology 143-160.

Lim, G.; Lee, M. L.; Hsu, W.; and Wong, T. Y. 2014. Transformed representations for convolutional neural networks in diabetic retinopathy screening. In AAAI Workshop on Modern Artificial Intelligence for Health Analytics (MAIHA), 34-38.

Lim, G.; Hsu, W.; and Lee, M. L. 2018. Intermediate goals in deep learning for retinal image analysis. In ACCV Workshop on AI for Retinal Image Analysis (AIRIA).
Litjens, G.; Kooi, T.; Bejnordi, B. E.; Setio, A. A. A.; Ciompi, F.; Ghafoorian, M.; van der Laak, J. A. W. M.; van Ginneken, B.; and Sanchez, C. I. 2017. A survey on deep learning in medical image analysis. Medical Image Analysis 42:60-88.

McGeechan, K.; Liew, G.; Macaskill, P.; Irwid, L.; Klein, R.; Klein, B. E. K.; Wang, J. J.; Mitchell, P.; Vingerling, J. R.; do Jong, P. T. V. M.; Witteman, J. C. M.; Breteler, M. M. B.; Shaw, J.; Zimmet, P.; and Wong, T. Y. 2009. Prediction of incident stroke events based on retinal vessel caliber: a systematic review and individual-participant meta-analysis. American Journal of Epidemiology 170(11):1323-1332.

Niemeijer, M.; van Ginneken, B.; Cree, M. J.; Mizutani, A.; Quellec, G.; Sanchez, C. I.; Zhang, B.; Hornero, R.; Lamard, M.; Muramatsu, C.; Wu, X.; Cazuguel, G.; You, J.; Mayo, A.; Li, Q.; Hatanaka, Y.; Cochener, B.; Roux, C.; Karray, F.; Garcia, M.; Fujita, H.; and Abramoff, M. D. 2010. Retinopathy online challenge: automatic detection of microaneurysms in digital color fundus photographs. IEEE Transactions on Medical Imaging 29(1):185-195.

Orobix. 2016. Retina blood vessel segmentation with a convolution neural network. https://github.com/orobix/retina-unet.

Poplin, R.; Varadarajan, A. V.; Blumer, K.; Liu, Y.; McConnell, M. V.; Corrado, G. S.; Peng, L.; and Webster, D. R. 2018. Prediction of cardiovascular risk factors from retinal fundus photographs via deep learning. Nature Biomedical Engineering 2(3):158.

Quiñonero-Candela, J.; Sugiyama, M.; Schwaighofer, A.; and Lawrence, N. D. 2009. Dataset Shift in Machine Learning. MIT Press.

Ronneberger, O.; Fischer, P.; and Brox, T. 2015. U-net: Convolutional networks for biomedical image segmentation. In MICCAI, 234-241.

Selvaraju, R. R.; Cogswell, M.; Das, A.; Vedantam, R.; Parikh, D.; and Batra, D. 2017. Grad-cam: Visual explanations from deep networks via gradient-based localization. In ICCV, 618-626.

Shortliffe, E. H., and Sepúlveda, M. J. 2018. Clinical decision support in the era of artificial intelligence. JAMA 320(21):21992200 .

Simonyan, K., and Zisserman, A. 2015. Very deep convolutional networks for large-scale image recognition. In ICLR.

Sun, B.; Feng, J.; and Saenko, K. 2016. Return of frustratingly easy domain adaptation. In AAAI, 2058-2065.

Ting, D. S. W.; Cheung, C. Y.; Lim, G.; Tan, G. S. W.; Quang, N. D.; Gan, A.; Hamzah, H.; Garcia-Franco, R.; San Yeo, I. Y.; Lee, S. Y.; Wong, E. Y. M.; Sabanayagam, C.; Baskaran, M.; Ibrahim, F.; Tan, N. C.; Finkelstein, E. A.; Lamoureux, E. L.; Wong, I. Y.; Bressler, N. M.; Sivaprasad, S.; Vama, R.; Jonas, J. B.; He, M. G.; Cheng, C. Y.; Cheung, G. C. M.; Aung, T.; Hsu, W.; Lee, M. L.; and Wong, T. Y. 2017. Development and validation of a deep learning system for diabetic retinopathy and related eye diseases using retinal images from multiethnic populations with diabetes. JAMA 318(22):2211-2223.

Trucco, E.; Ruggeri, A.; Karnowski, T.; Giancardo, L.; Chaum, E.; Hubschman, J. P.; Al-Diri, B.; Cheung, C. Y.; Wong, D.; Abramoff, M.; Lim, G.; Kumar, D.; Burlina, P.; Bressler, N. M.; Jelinek, H. F.; Meriaudeau, F.; Quellec, G.; Macgillivray, T.; and Dhillon, B. 2013. Validating retinal fundus image analysis algorithms: issues and a proposal. Investigative Ophthalmology \& Visual Science 54(5):3546-3559.

Xu, D.; Lim, G.; Lee, M. L.; and Hsu, W. 2018. A differentialbased approach for vessel type classification in retinal images. In ICIP, 2790-2794. IEEE. 